\title{
The Neumann Problem of Complex Special Lagrangian Equations with Supercritical Phase
}

\author{
Chuanqiang Chen ${ }^{1}$, Xinan $\mathrm{Ma}^{2, *}$ and Wei Wei ${ }^{2}$ \\ ${ }^{1}$ Department of Applied Mathematics, Zhejiang University of Technology, Hangzhou, \\ 310023, Zhejiang, China \\ 2 School of Mathematical Sciences, University of Science and Technology of China, \\ Hefei, 230026, Anhui, China
}

Received 22 August 2017; Accepted (in revised version) 8 June 2018

\begin{abstract}
Inspired by the Neumann problem of real special Lagrangian equations with supercritical phase, we consider the Neumann problem of complex special Lagrangian equations with supercritical phase in this paper, and establish the global $C^{2}$ estimates and the existence theorem by the method of continuity.
\end{abstract}

Key Words: Special Lagrangian equation, Neumann problem, supercritical phase.

AMS Subject Classifications: 35J60, 35B45

\section{Introduction}

As we all know, the real special Lagrangian equation is

$$
\arctan D^{2} u=\Theta(x),
$$

where

$$
\arctan D^{2} u:=\arctan \lambda_{1}+\arctan \lambda_{2}+\cdots+\arctan \lambda_{n},
$$

$\lambda=\left(\lambda_{1}, \lambda_{2}, \cdots, \lambda_{n}\right)$ are the eigenvalues of the Hessian matrix

$$
D^{2} u=\left\{\frac{\partial^{2} u}{\partial x_{i} \partial x_{j}}\right\}_{1 \leq i, j \leq n^{\prime}}
$$

and $\Theta$ is called the phase. In particular, $\Theta=\frac{(n-2) \pi}{2}$ is the critical phase, and if $\frac{(n-2) \pi}{2}<$ $\Theta(x)<\frac{n \pi}{2}$, Eq. (1.1) is called special Lagrangian equations with supercritical phase.

*Corresponding author. Email addresses: chuanqiangchen@zjut.edu.cn (C. Q. Chen), xinan@ustc.edu.cn (X. N. Ma), weisx001@mail.ustc.edu.cn (W. Wei) 
The special Lagrangian equation (1.1) was introduced by Harvey-Lawson [13] in the study of calibrated geometries. Here $\Theta$ is a constant called the phase angle. In this case the graph $x \mapsto(x, D u(x))$ defines a calibrated, minimal submanifold of $\mathbb{R}^{2 n}$. Since the work of Harvey-Lawson, special Lagrangian manifolds have gained wide interest, due in large part to their fundamental role in the Strominger-Yau-Zaslow description of mirror symmetry [26].

For the special Lagrangian equations with supercritical phase, Yuan obtained the interior $C^{1}$ estimate with Warren in [29] and the interior $C^{2}$ estimate with Wang in [28]. Recently Collins-Picard-Wu [8] obtained the existence theorem of the Dirichlet problem.

Moreover, for the Dirichlet problem of elliptic equations in $\mathbb{R}^{n}$, many results are known. For example, the Dirichlet problem of Laplace equation is studied in $[7,11]$, Caffarelli-Nirenberg-Spruck [2] and Ivochkina [15] solved the Dirichlet problem of Monge-Ampère equation, and Caffarelli-Nirenberg-Spruck [4] solved the Dirichlet problem of $k$-Hessian equation. After the pioneering works of Caffarelli et al., the Dirichlet problem of the general Hessian quotient equation was solved by Trudinger in [27]. For more information about the related subjects, we refer to the citations of $[2,4,15]$.

Also, the Neumann or oblique derivative problem of partial differential equations was widely studied. For a priori estimates and the existence theorem of Laplace equation with Neumann boundary condition, we refer to the book [11]. Also, we can see the recent book written by Lieberman [21] for the Neumann and the oblique derivative problems of linear and quasilinear elliptic equations. In 1986, Lions-Trudinger-Urbas solved the Neumann problem of Monge-Ampère equation in the celebrated paper [23]. Recently, MaQiu [24] solved the the Neumann problem of $k$-Hessian equations, and Chen-Zhang [6] generalized the result to the the Neumann problem of Hessian quotient equations. For the Neumann problem of special Lagrangian equations with supercritical phase, ChenMa-Wei [5] got the existence theorem. In [16, 17], Jiang-Trudinger studied the general oblique boundary value problems for augmented Hessian equations with some regular condition and some concavity condition.

At the same time, the complex equations have attracted a variety of mathematicians and many excellent works have been done. The complex Monge-Ampère equations are definitely one of the most important equations in partial differential equation and the geometry. In [1], Bedford and Taylor studied the Dirichlet problem of complex MongeAmpère equations by using the Perron-Bremermann family method, and got the existence and uniqueness of the weak solutions and a global Lipschitz regularity for plurisubharmonic solution when $\Omega$ is a bounded strictly pseudoconvex domain in $\mathbb{C}^{n}$. In [3], Caffarelli et al. studied the classical solution on strongly pseudoconvex domains. Their work was extended to arbitrary bounded domains in $\mathbb{C}^{n}$ by Guan in [12] under some subsolution condition. Recently, Fu-Yau equation on compact Kähler manifolds arises much attention, which was introduced in [10]. The Fu-Yau equation was solved in dimension 2 by Fu-Yau and was recently extended to higher dimensions in some cases in [25] by Phong, Picard and Zhang. In high dimensions, the equation is actually a 2-Hessian type equation. Complex Hessian equations have been studied extensively by many authors 
in recent years. On $\mathbb{C}^{n}$, the Dirichlet problem of complex Hessian equation was studied by $\mathrm{Li}$ [20] under some strict subsolution condition. On compact manifolds there were the existence of admissible solution in $[9,14]$. For the Neumann problem of complex Monge-Ampère, Li proved the existence under some other conditions in [19].

Naturally, we want to know how about the problem of complex special Lagrangian equations of the form

$$
\begin{cases}\arctan \partial \bar{\partial} u=\Theta(z) & \text { in } \Omega, \\ D_{v} u=-\varepsilon u+\varphi(z) & \text { on } \partial \Omega,\end{cases}
$$

where $\Omega$ is a strictly pseudo-convex domain in $\mathbb{C}^{n}, \varepsilon>0$ is constant, $v$ is the outer unit normal vector of $\partial \Omega, u$ is a $C^{2}$ and real valued function in $\Omega, \Theta$ is a real valued function in $\Omega, \partial \bar{\partial} u=\left\{\frac{\partial^{2} u}{\partial z_{i} \partial \bar{z}_{j}}\right\}_{1 \leq i, j \leq n}$, and $\arctan \partial \bar{\partial} u:=: \arctan \lambda_{1}+\arctan \lambda_{2}+\cdots+\arctan \lambda_{n}$, where $\lambda=$ $\left(\lambda_{1}, \lambda_{2}, \cdots, \lambda_{n}\right)$ are the eigenvalues of the complex Hessian matrix $\partial \bar{\partial} u$. In the following, we denote

$$
\begin{aligned}
& z=\left(z_{1}, \cdots, z_{n}\right) \in \bar{\Omega}, \quad z_{j}=t_{j}+\sqrt{-1} t_{n+j}, \quad t=\left(t_{1}, \cdots, t_{n}, t_{n+1}, \cdots, t_{2 n}\right), \\
& \partial_{j} u=\frac{\partial u}{\partial z_{j}}=u_{z_{j}}, \quad \partial_{j} u=\frac{\partial u}{\partial z_{\bar{j}}}=u_{z_{\bar{j}}}, \quad \partial u=\left(\partial_{1} u, \cdots, \partial_{n} u\right), \\
& D_{k} u=\frac{\partial u}{\partial t_{k}}, \quad D u=\left(D_{1} u, \cdots, D_{2 n} u\right),
\end{aligned}
$$

where $\sqrt{-1}$ is the imaginary unit. It is easy to know

$$
\begin{aligned}
& \partial_{j} u=\frac{1}{2}\left[D_{j} u-\sqrt{-1} D_{n+j} u\right], \quad|\partial u|^{2}=\sum_{j=1}^{n} \partial_{j} u \overline{\partial_{j} u}=\frac{1}{4}|D u|^{2} . \\
& \partial_{j j} u=\frac{1}{4}\left[D_{j j} u+D_{n+j n+j} u\right] .
\end{aligned}
$$

In this paper, we establish global $C^{2}$ estimate of the Neumann problem of special Lagrangian equations with supercritical phase and obtain the existence theorem as follows:

Theorem 1.1. Suppose $\Omega \subset \mathbb{C}^{n}$ is a $C^{4}$ strictly pseudoconvex domain and $\varphi \in C^{3}(\partial \Omega)$. Let $\Theta(z) \in C^{2}(\bar{\Omega})$ with $\frac{(n-2) \pi}{2}<\Theta(z)<\frac{n \pi}{2}$ in $\bar{\Omega}$. Moreover, if the domain $\Omega$ satisfies a geometric condition

$$
2 \kappa_{\min }+\varepsilon>0,
$$

where $\kappa_{\min }$ is the smallest principal curvature of $\partial \Omega$, then there exists a unique solution $u \in$ $C^{3, \alpha}(\bar{\Omega})$ of the Neumann problem of special Lagrangian equation (1.2).

As Li mentioned in his paper [19], because the Neumann boundary condition is not invariant under the holomorphic changes of variables, the strictly pesudoconvex domain may not be enough and we need the extra curvature condition of the domain.

Furthermore, we can obtain the existence theorem of the classical Neumann problem of complex special Lagrangian equation as below. 
Theorem 1.2. Suppose $\Omega \subset \mathbb{C}^{n}$ is a $C^{4}$ strictly convex domain and $v$ is outer unit normal vector of $\partial \Omega$. Let $\varphi \in C^{3}(\partial \Omega)$ and $\Theta(z) \in C^{2}(\bar{\Omega})$ with $\frac{(n-2) \pi}{2}<\Theta(z)<\frac{n \pi}{2}$ in $\bar{\Omega}$. Then there exists a unique constant $\beta$ such that the Neumann problem of special Lagrangian equation

$$
\begin{cases}\arctan \partial \bar{\partial} u=\Theta(z) & \text { in } \Omega, \\ D_{v} u=\beta+\varphi(z) & \text { on } \partial \Omega,\end{cases}
$$

has admissible solutions $u \in C^{3, \alpha}(\bar{\Omega})$, which are unique up to a constant.

The rest of the paper is organized as follows. In Section 2, we collect some properties of the special Lagrangian equation and establish the $C^{0}$ estimate for the Neumann problem of special Lagrangian equation. The $C^{1}$ and $C^{2}$ estimates are established in Section 3 , Section 4, respectively. At last, we prove Theorem 1.1 and Theorem 1.2 in Section 5.

\section{Some properties and $C^{0}$ estimate}

In this section, we give some properties of the special Lagrangian equation with supercritical phase and establish the $C^{0}$ estimate.

\subsection{Some properties}

Property 2.1. Suppose $\Omega \subset \mathbb{C}^{n}$ is a domain and $\Theta(z) \in C^{0}(\bar{\Omega})$ with $\frac{(n-2) \pi}{2}<\Theta(z)<\frac{n \pi}{2}$ in $\bar{\Omega}$. Let $u \in C^{2}(\Omega)$ be a solution of special Lagrangian equation

$$
\arctan \partial \bar{\partial} u=\Theta(z) \text { in } \Omega \text {. }
$$

We assume $\lambda=\left(\lambda_{1}, \lambda_{2}, \cdots, \lambda_{n}\right)$ are the eigenvalues of the Hessian matrix $\partial \bar{\partial} u$ with $\lambda_{1} \geq$ $\lambda_{2} \cdots \geq \lambda_{n}$, then we have some properties:

$$
\begin{aligned}
& \lambda_{1} \geq \cdots \geq \lambda_{n-1}>0, \\
& \left|\lambda_{n}\right| \leq \lambda_{n-1} \\
& \sum_{i=1}^{n} \lambda_{i}>0 \\
& \left|\lambda_{n}\right|<C_{0}
\end{aligned}
$$

where

$$
C_{0}=\max \left\{\tan \left(\frac{(n-1) \pi}{2}-\min _{\bar{\Omega}} \Theta\right), \tan \left(\frac{1}{n} \max _{\bar{\Omega}} \Theta\right)\right\} .
$$

Proof. For any $i=1,2, \cdots, n$, we know $\arctan \lambda_{i} \in\left(-\frac{\pi}{2}, \frac{\pi}{2}\right)$. Then we can get

$$
\begin{aligned}
\arctan \lambda_{n-1}+\arctan \lambda_{n} & =\Theta-\arctan \lambda_{1}-\arctan \lambda_{2} \cdots-\arctan \lambda_{n-2} \\
& \geq \Theta-\frac{(n-2) \pi}{2}>0 .
\end{aligned}
$$


So $\lambda_{n-1}+\lambda_{n}>0$, which implies (2.2a), (2.2b) and (2.2c) hold.

Moreover,

$$
\arctan \lambda_{n}=\Theta-\arctan \lambda_{1}-\arctan \lambda_{2}-\cdots-\arctan \lambda_{n-1}>\min _{\bar{\Omega}} \Theta-\frac{(n-1) \pi}{2}
$$

and

$$
\arctan \lambda_{n}<\frac{\arctan \lambda_{1}+\arctan \lambda_{2}+\cdots+\arctan \lambda_{n}}{n} \leq \frac{1}{n} \max _{\bar{\Omega}} \Theta
$$

so we can get

$$
\left|\lambda_{n}\right|<\max \left\{\tan \left(\frac{(n-1) \pi}{2}-\min _{\bar{\Omega}} \Theta\right), \tan \left(\frac{1}{n} \max _{\bar{\Omega}} \Theta\right)\right\} .
$$

Thus, we complete the proof.

Property 2.2. Suppose $\Omega \subset \mathbb{C}^{n}$ is a domain and $\Theta(z) \in C^{2}(\bar{\Omega})$ with $\frac{(n-2) \pi}{2}<\Theta(z)<\frac{n \pi}{2}$ in $\bar{\Omega}$. Let $u \in C^{4}(\Omega)$ be a solution of complex special Lagrangian equation (2.1). Then for any $\xi \in \mathbb{S}^{2 n-1}$, we have

$$
\sum_{i j=1}^{n} F^{i \bar{j}} D_{\xi \xi \xi} \partial_{i \bar{j}} u \geq D_{\xi \xi} \Theta-A\left|D_{\tilde{\zeta}} \Theta\right|^{2} \quad \text { in } \Omega,
$$

where

$$
F^{i \bar{j}}=\frac{\partial \arctan \partial \bar{\partial} u}{\partial u_{i \bar{j}}} \quad \text { and } \quad A=\frac{2}{\tan \left(\min _{\bar{\Omega}} \Theta-\frac{(n-2) \pi}{2}\right)} .
$$

Proof. For any $z \in \Omega$, we can assume $\partial \bar{\partial} u$ is diagonal with $\lambda_{i}=u_{i \bar{i}}$, since (2.3) is invariant under rotating the coordinates. Then we have

$$
F^{i \bar{j}}=: \frac{\partial \arctan \partial \bar{\partial} u}{\partial u_{i \bar{j}}}= \begin{cases}\frac{1}{1+\lambda_{i}^{2}}, & \text { if } i=j, \\ 0, & \text { if } i \neq j,\end{cases}
$$

and

$$
F^{i j, k \bar{l}}=: \frac{\partial^{2} \arctan \partial \bar{\partial} u}{\partial u_{i j} \partial u_{k \bar{l}}}= \begin{cases}-\frac{2 \lambda_{i}}{\left(1+\lambda_{i}^{2}\right)^{2}}, & \text { if } i=j=k=l, \\ -\frac{\lambda_{i}+\lambda_{j}}{\left(1+\lambda_{i}^{2}\right)\left(1+\lambda_{j}^{2}\right)}, & \text { if } i=l, \quad j=k, \quad i \neq j, \\ 0, & \text { otherwise. }\end{cases}
$$


From the complex special Lagrangian equation (2.1), we know

$$
\sum_{i j=1}^{n} F^{i \bar{j}} D_{\xi} \partial_{i j} u=D_{\xi} \Theta
$$

and

$$
\begin{aligned}
\sum_{i j=1}^{n} F^{i \bar{j}} D_{\xi \xi} \partial_{i \bar{j}} u & =D_{\xi \xi} \Theta-\sum_{i j k l=1}^{n} F^{i \bar{j}, k \bar{l}} D_{\xi} \partial_{i \bar{j}} u D_{\xi} \partial_{k \bar{l}} u \\
& =D_{\xi \xi} \Theta-\sum_{i=1}^{n} F^{i \bar{i}, i \bar{i}}\left(D_{\xi} \partial_{i \bar{i}} u\right)^{2}-\sum_{i \neq j} F^{i \bar{j}, j \bar{i}}\left|D_{\xi} \partial_{i \bar{j}} u\right|^{2} \\
& \geq D_{\xi \xi} \Theta-\sum_{i=1}^{n} F^{i \bar{i}, i \bar{i}}\left(D_{\xi} \partial_{i \bar{i}} u\right)^{2} .
\end{aligned}
$$

From the concavity lemma (Lemma 2.2 in [8]), we know

$$
\begin{aligned}
-\sum_{i=1}^{n} F^{i \bar{i}, i \bar{i}}\left(D_{\xi} \partial_{i \bar{i}} u\right)^{2} & \geq-\frac{2}{\tan \left(\min _{\bar{\Omega}} \Theta-\frac{(n-2) \pi}{2}\right)}\left(\sum_{i=1}^{n} F^{i \bar{i}} D_{\tilde{\zeta}} \partial_{i \bar{i}} u\right)^{2} \\
& =-\frac{2}{\tan \left(\min _{\bar{\Omega}} \Theta-\frac{(n-2) \pi}{2}\right)}\left|D_{\tilde{\xi}} \Theta\right|^{2} .
\end{aligned}
$$

Hence (2.3) holds.

\section{$2.2 C^{0}$ estimate}

The $C^{0}$ estimate is easy. For completeness, we produce a proof here following the idea of Lions-Trudinger-Urbas [23] and Ma-Qiu [24].

Theorem 2.3. Suppose $\Omega \subset \mathbb{C}^{n}$ is a $C^{1}$ bounded domain and $\varphi \in C^{0}(\partial \Omega)$. Let $\Theta(z) \in C^{0}(\bar{\Omega})$ with $\frac{(n-2) \pi}{2}<\Theta(z)<\frac{n \pi}{2}$ in $\bar{\Omega}$ and $u \in C^{2}(\Omega) \cap C^{1}(\bar{\Omega})$ be the solution of complex special Lagrangian equation (1.2), then we have

$$
-M_{0} \leq u(z) \leq \frac{1}{\varepsilon} \max _{\partial \Omega}|\varphi| \quad \text { in } \Omega,
$$

where

$$
M_{0}=\frac{1}{\varepsilon} \max _{\partial \Omega}|\varphi|+\frac{2 B}{\varepsilon} \operatorname{diam}(\Omega)+B \operatorname{diam}(\Omega)^{2} \quad \text { and } \quad B=\tan \left(\frac{1}{n} \max _{\bar{\Omega}} \Theta\right)<+\infty .
$$

Proof. From (2.2c), we know $u$ is subharmonic. So the maximum of $u$ is attained at a boundary point $z_{0} \in \partial \Omega$. Then we can get

$$
0 \leq D_{v} u\left(z_{0}\right)=-\varepsilon u\left(z_{0}\right)+\varphi\left(z_{0}\right) .
$$


Hence

$$
\max _{\bar{\Omega}} u=u\left(z_{0}\right) \leq \frac{\varphi\left(z_{0}\right)}{\varepsilon} \leq \frac{1}{\varepsilon} \max _{\partial \Omega}|\varphi|
$$

Without loss of generality, we assume $0 \in \Omega$ and denote

$$
B=\tan \left(\frac{1}{n} \max _{\bar{\Omega}} \Theta\right)<+\infty
$$

Then we have

$$
\arctan \partial \bar{\partial} u=\Theta \leq \max _{\bar{\Omega}} \Theta=\arctan \partial \bar{\partial}\left(B|z|^{2}\right)
$$

By the comparison principle, we know $u-B|z|^{2}$ attains its minimum at a boundary point $z_{1} \in \partial \Omega$. Then

$$
\begin{aligned}
0 & \geq\left. D_{v}\left(u-B|z|^{2}\right)\right|_{z=z_{1}}=D_{v} u\left(z_{1}\right)-\left.B D_{v}\left(|z|^{2}\right)\right|_{z=z_{1}} \\
& =-\varepsilon u\left(z_{1}\right)+\varphi\left(z_{1}\right)-\left.2 B \cdot t \cdot v\right|_{z=z_{1}} \\
& \geq-\varepsilon u\left(z_{1}\right)-\max _{\partial \Omega}|\varphi|-2 B \operatorname{diam}(\Omega) .
\end{aligned}
$$

Hence

$$
\begin{aligned}
\min _{\bar{\Omega}} u & \geq \min _{\bar{\Omega}}\left(u-B|z|^{2}\right)=u\left(z_{1}\right)-B\left|z_{1}\right|^{2} \\
& \geq-\frac{1}{\varepsilon} \max _{\partial \Omega}|\varphi|-\frac{2 B}{\varepsilon} \operatorname{diam}(\Omega)-B \operatorname{diam}(\Omega)^{2} .
\end{aligned}
$$

Thus, we complete the proof.

\section{Global gradient estimate}

In this section, we prove the global gradient estimate by following the idea of Li [19].

Theorem 3.1. Suppose $\Omega \subset \mathbb{C}^{n}$ is a $C^{3}$ bounded domain satisfying (1.3) and $\varphi \in C^{2}(\partial \Omega)$. Let $\Theta(z) \in C^{1}(\bar{\Omega})$ with $\frac{(n-2) \pi}{2}<\Theta(z)<\frac{n \pi}{2}$ in $\bar{\Omega}$ and $u \in C^{3}(\Omega) \cap C^{2}(\bar{\Omega})$ be a solution of complex special Lagrangian equation (1.2), then we have

$$
\sup _{\bar{\Omega}}|D u| \leq M_{1}
$$

where $M_{1}$ depends on $n, \Omega, \kappa_{\min }+\varepsilon,|u|_{C^{0}}, \max _{\bar{\Omega}} \Theta, \min _{\bar{\Omega}} \Theta$ and $|\varphi|_{C^{2}}$. 
Proof. In order to prove (3.1), it suffices to prove

$$
D_{\tilde{\xi}} u(z) \leq M_{1}, \quad \forall(z, \xi) \in \bar{\Omega} \times \mathbb{S}^{2 n-1} .
$$

For any $(z, \xi) \in \bar{\Omega} \times \mathbb{S}^{2 n-1}$, denote

$$
W(z, \xi)=D_{\xi} u(z)-\langle v, \xi\rangle(-\varepsilon u+\varphi(z))+K_{0} u^{2}+K_{1}|z|^{2},
$$

where $K_{0}$ and $K_{1}$ are large constants to be determined later, and $v$ is a $C^{2}(\bar{\Omega})$ extension of the outer unit normal vector field on $\partial \Omega$. We still use $\varphi(z)$ to denote a $C^{2}$ extension of $\varphi$ from $\partial \Omega$ to $\Omega$. Assume $W$ achieves its maximum at $\left(z_{0}, \xi_{0}\right) \in \bar{\Omega} \times \mathrm{S}^{2 n-1}$. It is easy to know $D_{\tilde{\zeta}_{0}} u\left(z_{0}\right)>0$. We claim $z_{0} \in \partial \Omega$. Otherwise, if $z_{0} \in \Omega$, we rotate the coordinates such that $\left\{\partial_{i j} u\left(z_{0}\right)\right\}$ is diagonal with $\lambda_{i}=u_{i \bar{i}}$ and $\lambda_{1} \geq \lambda_{2} \geq \cdots \geq \lambda_{n}$. Then we have

$$
F^{i \bar{j}}=: \frac{\partial \arctan \partial \bar{\partial} u}{\partial u_{i \bar{j}}}= \begin{cases}\frac{1}{1+\lambda_{i}^{2}}, & \text { if } i=j, \\ 0, & \text { if } i \neq j .\end{cases}
$$

Hence we can get from Property 2.1

$$
\begin{aligned}
& F^{1 \overline{1}} \leq F^{2 \overline{2}} \leq \cdots \leq F^{n \bar{n}}, \\
& F^{n \bar{n}}=\frac{1}{1+\lambda_{n}^{2}} \geq c_{0}>0, \\
& F^{i \bar{i}} u_{i \bar{i}}=\frac{\lambda_{i}}{1+\lambda_{i}^{2}} \in\left(-\frac{1}{2}, \frac{1}{2}\right),
\end{aligned}
$$

where

$$
c_{0}=\frac{1}{1+\max \left\{\tan \left(\frac{(n-1) \pi}{2}-\min _{\bar{\Omega}} \Theta\right), \tan \left(\frac{1}{n} \max _{\bar{\Omega}} \Theta\right)\right\}^{2}} .
$$

For fixed $\xi=\xi_{0}, W\left(z, \xi_{0}\right)$ achieves its maximum at the same point $z_{0} \in \Omega$ and we can easily get that at $z_{0}$,

$$
\begin{aligned}
0 \geq F^{i \bar{j}} \partial_{i \bar{j}} W= & F^{i \bar{i}}\left[\partial_{i \bar{i}} D_{\tilde{\zeta}_{0}} u-\left\langle v, \xi_{0}\right\rangle_{i \bar{i}}(-\varepsilon u+\varphi)-\left\langle v, \xi_{0}\right\rangle\left(-\varepsilon u_{i \bar{i}}+\varphi_{i \bar{i}}\right)\right. \\
& -\left\langle v, \xi_{0}\right\rangle_{i}\left(-\varepsilon u_{\bar{i}}+\varphi_{\bar{i}}\right)-\left\langle v, \xi_{0}\right\rangle_{\bar{i}}\left(-\varepsilon u_{i}+\varphi_{i}\right) \\
& \left.+2 K_{0} u_{i} u_{\bar{i}}+2 K_{0} u u_{i \bar{i}}+K_{1}\right] \\
\geq & D_{\tilde{\zeta}_{0}} \Theta+\left(2 K_{0}-C_{1}\right) \sum_{i=1}^{n} F^{i \bar{i}} u_{i} u_{\bar{i}} \\
+ & {\left[2 K_{0} u+\varepsilon\left\langle v, \xi_{0}\right\rangle\right] \sum_{i=1}^{n} F^{i \bar{i}} u_{i \bar{i}}+\left(K_{1}-C_{1}\right) \sum_{i=1}^{n} F^{i \bar{i}}, }
\end{aligned}
$$

where $C_{1}$ is a positive constant depending only on $n, \varepsilon,|u|_{C^{0}},|\varphi|_{C^{2}}$ and $|v|_{C^{2}}$. 
Take $K_{0}=\frac{C_{1}}{2}$ and $K_{1}=2 C_{1}+\frac{2|D \Theta|+n\left[2 K_{0}|u|+\varepsilon\right]}{c_{0}}$. Using

$$
\sum_{i=1}^{n} F^{i \bar{i}} u_{i \bar{i}} \in\left(-\frac{n}{2}, \frac{n}{2}\right), \quad \sum_{i=1}^{n} F^{i \bar{i}}>F^{n \bar{n}} \geq c_{0}
$$

we can get

$$
\begin{aligned}
0 & \geq F^{i \bar{j}} \partial_{i j} W \\
& \geq-\left|D_{\xi_{0}} \Theta\right|-\frac{n}{2}\left[2 K_{0}|u|+\varepsilon\right]+\left(2 K_{0}-C_{1}\right) \sum_{i=1}^{n} F^{i \bar{i}} u_{i} u_{\bar{i}}+\left(K_{1}-C_{1}\right) \sum_{i=1}^{n} F^{i \bar{i}} \\
& >0 .
\end{aligned}
$$

This is a contradiction. So $z_{0} \in \partial \Omega$. Then we continue our proof in the following three cases.

(a) If $\xi_{0}$ is normal at $z_{0} \in \partial \Omega$, then

$$
W\left(z_{0}, \xi_{0}\right)=K_{0} u^{2}+K_{1}\left|z_{0}\right|^{2} \leq C_{2} .
$$

Then we can easily get (3.2).

(b) If $\xi_{0}$ is non-tangential at $z_{0} \in \partial \Omega$, then we can write $\xi_{0}=\alpha \tau+\beta v$, where $\tau \in \mathbb{S}^{2 n-1}$ is tangential at $z_{0}$, that is $\langle\tau, v\rangle=0, \alpha=\left\langle\xi_{0}, \tau\right\rangle>0, \beta=\left\langle\xi_{0}, v\right\rangle<1$, and $\alpha^{2}+\beta^{2}=1$. Then we have

$$
\begin{aligned}
W\left(z_{0}, \xi_{0}\right) & =\beta D_{\tau} u+K_{0} u^{2}+K_{1}\left|z_{0}\right|^{2} \\
& \leq \beta W\left(z_{0}, \xi_{0}\right)+(1-\beta)\left(K_{0} u^{2}+K_{1}\left|z_{0}\right|^{2}\right),
\end{aligned}
$$

so

$$
W\left(z_{0}, \xi_{0}\right) \leq K_{0} u^{2}+K_{1}\left|z_{0}\right|^{2} \leq C_{3} .
$$

Then we can easily get (3.2).

(c) If $\xi_{0}$ is tangential at $z_{0} \in \partial \Omega$, we may assume that the outer normal direction of $\Omega$ at $z_{0}$ is $(0, \cdots, 0,1)$. By a rotation, we assume that $\xi_{0}=(1, \cdots, 0)=e_{1}$. Then we have

$$
\begin{aligned}
0 & \leq D_{v} W\left(z_{0}, \xi_{0}\right)=D_{v} D_{1} u-D_{v}\left\langle v, \xi_{0}\right\rangle(-\varepsilon u+\varphi)+2 K_{0} u \cdot D_{v} u+K_{1} D_{v}|z|^{2} \\
& \leq D_{v} D_{1} u+C_{4} \\
& =D_{1} D_{v} u-D_{1} v_{k} D_{k} u+C_{4} .
\end{aligned}
$$

By the boundary condition, we know

$$
D_{1} D_{v} u=D_{1}(-\varepsilon u+\varphi) \leq-\varepsilon W\left(z_{0}, \xi_{0}\right)+C_{5} .
$$


Following the argument of [19], we can get

$$
-D_{1} v_{k} D_{k} u \leq-\kappa_{\min } W\left(z_{0}, \xi_{0}\right)+C_{6} .
$$

From (1.3), it holds $\kappa_{\min }+\varepsilon>0$. So

$$
W\left(z_{0}, \xi_{0}\right) \leq \frac{C_{4}+C_{5}+C_{6}}{\kappa_{\min }+\varepsilon}
$$

Then we can easily get (3.2).

Remark 3.1. To prove Theorem 1.2, we need to consider the complex special Lagrangian equation (1.2) with $\varepsilon \rightarrow 0$. Under the assumptions of Theorem 1.2, that is, $\Omega \subset \mathbb{C}^{n}$ is a $C^{4}$ strictly convex domain, $\varphi \in C^{3}(\partial \Omega), \Theta(z) \in C^{2}(\bar{\Omega})$ and $\frac{(n-2) \pi}{2}<\Theta(z)<\frac{n \pi}{2}$ in $\bar{\Omega}$, and for any sufficiently small $\varepsilon$, we can obtain the following proof of Theorem 2.3

$$
\sup _{\bar{\Omega}} \varepsilon\left|u^{\varepsilon}\right| \leq \widetilde{M}_{0}
$$

for the solution $u^{\varepsilon}$ of (1.2), where $\widetilde{M}_{0}$ depends on $n, \operatorname{diam}(\Omega)$, $\max _{\bar{\Omega}} \Theta$, and $|\varphi|_{C^{0}}$. Moreover, we can choose test function

$$
W(z, \xi)=D_{\xi} u^{\varepsilon}(z)-\langle v, \xi\rangle\left(-\varepsilon u^{\varepsilon}+\varphi(z)\right)+K_{0}\left(\varepsilon u^{\varepsilon}\right)^{2}+K_{1}|z|^{2},
$$

and following the proof of Theorem 3.1, we can choose $K_{0}$ and $K_{1}$ independent of $\varepsilon$, and obtain the global gradient estimate

$$
\sup _{\bar{\Omega}}\left|D u^{\varepsilon}\right| \leq \widetilde{M_{1}}
$$

where $\widetilde{M}_{1}$ depends on $n, \Omega, \max _{\bar{\Omega}} \Theta, \min _{\bar{\Omega}} \Theta,|\Theta|_{C^{1}}$ and $|\varphi|_{C^{2}}$, and is independent of $\varepsilon$. It is easy to know

$$
\sup _{\bar{\Omega}}\left|u^{\varepsilon}-\frac{1}{|\Omega|} \int_{\Omega} u^{\varepsilon}\right| \leq \widetilde{M}_{1}
$$

\section{Global second derivatives estimate}

We now come to the a priori estimate of global second derivatives. Firstly, we prove the estimate of double normal second derivatives on boundary, and then we complete the proof of global second derivatives estimate. 


\subsection{Estimate of double normal second derivatives on boundary}

Theorem 4.1. Suppose $\Omega \subset \mathbb{C}^{n}$ is a $C^{4}$ strictly pseudoconvex domain and $\varphi \in C^{3}(\partial \Omega)$. Let $\Theta(z) \in C^{2}(\bar{\Omega})$ with $\frac{(n-2) \pi}{2}<\Theta(z)<\frac{n \pi}{2}$ in $\bar{\Omega}$ and $u \in C^{4}(\Omega) \cap C^{3}(\bar{\Omega})$ be the solution of complex special Lagrangian equation (1.2), then we have

$$
\max _{\partial \Omega}\left|D_{v v} u\right| \leq \overline{M_{2}}
$$

where $\overline{M_{2}}$ depends on $n, \Omega, \max _{\bar{\Omega}} \Theta,|u|_{C^{1}}, \min _{\bar{\Omega}} \Theta,|\Theta|_{C^{2}}$ and $|\varphi|_{C^{3}}$.

Proof. Since $\Omega$ is a $C^{4}$ strictly pseudoconvex domain, there is a strictly plurisubharmonic defining function $r \in C^{4}(\bar{\Omega})$ such that

$$
\begin{array}{ll}
|D r|=1 & \text { on } \partial \Omega, \\
\partial \bar{\partial} r \geq k_{0} I_{n} & \text { in } \bar{\Omega},
\end{array}
$$

where $k_{0}$ is a positive constant depending only on $\Omega$ and $I_{n}$ is the $n \times n$ identity matrix.

Let $z_{0} \in \partial \Omega$ be an arbitrary point. By a shift and a rotation of the coordinates $\left\{z_{1}, \cdots, z_{n}\right\}$, we can assume that $z_{0}=0, \partial_{z_{i}} r(0)=0$ for $i<n$, and $D_{t_{n}} r(0)=-1, D_{t_{2 n}} r(0)=0$. In $\bar{B}(0, \delta) \cap \bar{\Omega}$, a sufficiently small neighborhood of $z_{0}$, we can get by the Taylor expansion of $r$ up to second order

$$
r(z)=-\operatorname{Re}\left(z_{n}-\sum_{i j=1}^{n} a_{i j} z_{i} z_{j}\right)+\sum_{i j=1}^{n} b_{i j} z_{i} \overline{z_{j}}+\mathcal{O}\left(|z|^{3}\right),
$$

where $\left\{b_{i j}\right\}=\partial \bar{\partial} r(0)$ is positive definite. We now introduce new coordinates $z^{\prime}=\psi(z)$ of the form

$$
z_{i}^{\prime}=z_{i} \quad \text { for } \quad i<n, \quad z_{n}^{\prime}=z_{n}-\sum_{i j=1}^{n} a_{i j} z_{i} z_{j} .
$$

In $\psi(\bar{B}(0, \delta) \cap \bar{\Omega})$, we have

$$
\left.r(z)\right|_{z=\psi^{-1}\left(z^{\prime}\right)}=-\operatorname{Re} z_{n}^{\prime}+\sum_{i j=1}^{n} b_{i j} z_{i}^{\prime} \overline{z_{j}^{\prime}}+\mathcal{O}\left(\left|z^{\prime}\right|^{3}\right),
$$

Denote

$$
\begin{aligned}
& r_{0}\left(z^{\prime}\right)=-\operatorname{Re} z_{n}^{\prime}+\sum_{i j=1}^{n} b_{i j} z_{i}^{\prime} \overline{z_{j}^{\prime}} \\
& B_{j}\left(z^{\prime}\right)=\left.\left(\sum_{i=1}^{n}\left[a_{i j}+a_{j i}\right] z_{i}\right)\right|_{z=\psi^{-1}\left(z^{\prime}\right)^{\prime}} \quad j=1, \cdots, n, \\
& A_{j}\left(z^{\prime}\right)=B_{j}\left(1-B_{n}\right)^{-1} \frac{\partial r_{0}\left(z^{\prime}\right)}{\partial \overline{z_{n}^{\prime}}}, \quad j=1, \cdots, n-1 .
\end{aligned}
$$


It is easy to know $\left|B_{j}\right|=\mathcal{O}\left(\left|z^{\prime}\right|\right)$ for $j=1, \cdots, n$, and $A_{j}$ is holomorphic in $z^{\prime} \in \psi(\bar{B}(0, \delta) \bigcap \bar{\Omega})$. Following the calculations in [19], we know the Neumann boundary condition in $z^{\prime}$ coordinates

$$
4 \operatorname{Re}\left(\left\langle\partial_{z^{\prime}} u, \partial_{z^{\prime}} r_{0}\right\rangle-\sum_{j=1}^{n-1} A_{j} \partial_{z_{j}^{\prime}} u\right)=\phi\left(z^{\prime}, u\right)+\mathcal{O}\left(\left|z^{\prime}\right|^{3}\right),
$$

where

$$
\phi\left(z^{\prime}, u\right)=\left.\left|1-B_{n}\left(z^{\prime}\right)\right|^{-2}(-\varepsilon u+\varphi(z))\right|_{z=\psi^{-1}\left(z^{\prime}\right)} .
$$

Following the idea of [19], we choose the auxiliary function

$$
h\left(z^{\prime}\right)=4 \operatorname{Re}\left[\left\langle\partial_{z^{\prime}} u, \partial_{z^{\prime}} r_{0}\right\rangle-\sum_{j=1}^{n-1} A_{j} \partial_{z_{j}^{\prime}} u\right]-\phi\left(z^{\prime}, u\right)+\left.K r(z)\right|_{z=\psi^{-1}\left(z^{\prime}\right)}-K_{1} \operatorname{Re}\left(z_{n}^{\prime}\right),
$$

where $K_{1}>0$ is sufficiently large so that

$$
h<0 \text { on } \psi(\partial(B(0, \delta))-\partial \Omega),
$$

and

$$
h=-K_{1} \operatorname{Re}\left(z_{n}^{\prime}\right)+\mathcal{O}\left(\left|z^{\prime}\right|^{2}\right) \leq 0 \quad \text { on } \psi(\partial(B(0, \delta)) \cap \partial \Omega) .
$$

Let

$$
F^{i \bar{j}}=\frac{\partial \arctan \partial_{z} \overline{\partial_{z}} u}{\partial u_{z_{i} \overline{z_{j}}}}, \quad G^{i \bar{j}}=\frac{\partial \arctan \partial_{z} \overline{\partial_{z}} u}{\partial u_{z_{i}^{\prime} \bar{z}_{j}^{\prime}}}
$$

It is easy to know

$$
G^{i \bar{j}}=F^{p \bar{q}}\left(\frac{\partial z_{i}^{\prime}}{\partial z_{p}} \overline{\left(\frac{\partial z_{j}^{\prime}}{\partial z_{q}}\right)} .\right.
$$

For any $z^{\prime} \in \psi(B(0, \delta) \cap \Omega)$, we can get

$$
\begin{aligned}
& G^{i \bar{j}} \partial_{z_{i}^{\prime} \bar{z}_{j}^{\prime}} h=2 G^{i \bar{j}} \partial_{z_{i}^{\prime} \bar{z}_{j}^{\prime}}\left(\partial_{z_{k}^{\prime}} u \partial_{\overline{z_{k}^{\prime}}} r_{0}+\partial_{\overline{z_{k}^{\prime}}} u \partial_{z_{k}^{\prime}} r_{0}\right)-2 G^{i \bar{j}} \partial_{z_{i}^{\prime} \bar{z}_{j}^{\prime}}\left(\sum_{k=1}^{n-1} A_{k} \partial_{z_{k}^{\prime}} u+\overline{A_{k}} \partial_{\overline{z_{k}^{\prime}}} u\right) \\
& -G^{i \bar{j}} \partial_{z_{i}^{\prime} \bar{z}_{j}^{\prime}} \phi+K G^{i \bar{j}} \partial_{z_{i}^{\prime} \bar{z}_{j}^{\prime}} r \\
& =2 G^{i \bar{j}}\left(\partial_{z_{k}^{\prime}} u_{z_{i}^{\prime} \overline{z_{j}^{\prime}}} \partial_{\overline{z_{k}^{\prime}}} r_{0}+\partial_{\overline{z_{k}^{\prime}}} u_{z_{i}^{\prime} \bar{z}_{j}^{\prime}} \partial_{z_{k}^{\prime}} r_{0}+u_{z_{k}^{\prime} \overline{z_{j}^{\prime}}} \partial_{z_{i}^{\prime} \overline{z_{k}^{\prime}}} r_{0}+u_{\overline{z_{k}^{\prime}} z_{i}^{\prime}} \partial_{z_{k}^{\prime} \overline{z_{j}^{\prime}}} r_{0}\right) \\
& -2 \sum_{k=1}^{n-1} G^{i \bar{j}}\left(A_{k} \partial_{z_{k}^{\prime}} u_{z_{i}^{\prime} \bar{z}_{j}^{\prime}}+\overline{A_{k}} \partial_{\overline{z_{k}^{\prime}}} u_{z_{i}^{\prime} \bar{z}_{j}^{\prime}}+\partial_{z_{i}^{\prime}} A_{k} u_{z_{k}^{\prime} \bar{z}_{j}^{\prime}}+\overline{\partial_{z_{j}^{\prime}} A_{k}} u_{z_{i}^{\prime} \bar{z}_{k}^{\prime}}\right) \\
& -G^{i \bar{j}}\left[\left|1-B_{n}\left(z^{\prime}\right)\right|^{-2}\left(-\varepsilon u_{z_{i}^{\prime} z_{j}^{\prime}}+\varphi_{z_{i}^{\prime} z_{j}^{\prime}}\right)+\partial_{z_{i}^{\prime} \overline{z_{j}^{\prime}}}\left(\left|1-B_{n}\left(z^{\prime}\right)\right|^{-2}\right)(-\varepsilon u+\varphi)\right] \\
& +K G^{i j} \partial_{z_{i}^{\prime} z_{j}^{\prime}}
\end{aligned}
$$




$$
\begin{aligned}
= & 2\left[\partial_{z_{k}^{\prime}} \Theta \partial_{\overline{z_{k}^{\prime}}} r_{0}+\partial_{\overline{z_{k}^{\prime}}} \Theta \partial_{z_{k}^{\prime}} r_{0}\right]+2 F^{p \bar{q}}\left(u_{z_{k}^{\prime} \overline{z_{q}}} \partial_{z_{p} \bar{z}_{k}} r_{0}+u_{\overline{z_{k}^{\prime}} z_{p}} \partial_{z_{k}^{\prime} \overline{z_{q}}} r_{0}\right) \\
& -2 \sum_{k=1}^{n-1}\left[A_{k} \partial_{z_{k}^{\prime}} \Theta+\overline{A_{k}} \partial_{\overline{z_{k}^{\prime}}} \Theta+F^{p \bar{q}}\left(\partial_{z_{p}} A_{k} u_{z_{k}^{\prime} \overline{z_{q}}}+\overline{\partial_{z_{q}}} A_{k} u_{z_{p} \overline{z_{k}^{\prime}}}\right)\right] \\
& -F^{p \bar{q}}\left[\left|1-B_{n}\left(z^{\prime}\right)\right|^{-2}\left(-\varepsilon u_{z_{p} \overline{z_{q}}}+\varphi_{z_{p} \overline{z_{q}}}\right)+\partial_{z_{p} \overline{z_{q}}}\left(\left|1-B_{n}\left(z^{\prime}\right)\right|^{-2}\right)(-\varepsilon u+\varphi)\right] \\
& +K F^{p \bar{q}} \partial_{z_{p} \overline{z_{q}}} r \\
\geq & K k_{0} \sum_{p=1}^{n} F^{p \bar{p}}-C_{7},
\end{aligned}
$$

where $F^{p \bar{q}} u_{z_{k}^{\prime} \bar{z}_{q}}=F^{p \bar{q}} u_{z_{m}} \overline{z_{q}} \frac{\partial z_{m}}{\partial z_{k}^{\prime}}$ and $F^{p \bar{q}} u_{z_{p} \bar{z}_{k}^{\prime}}=F^{p \bar{q}} u_{z_{p} \overline{z_{m}}} \overline{\left(\frac{\partial z_{m}}{\partial z_{k}^{\prime}}\right)}$ are bounded by rotating the coordinates $\left\{z_{1}, \cdots, z_{n}\right\}$ such that $\partial_{z} \overline{\partial_{z}} u$ is diagonal. From (2.2d), we know

$$
\sum_{p=1}^{n} F^{p \bar{p}} \geq c_{0}
$$

where

$$
c_{0}=\frac{1}{1+\max \left\{\tan \left(\frac{(n-1) \pi}{2}-\min _{\bar{\Omega}} \Theta\right), \tan \left(\frac{1}{n} \max _{\bar{\Omega}} \Theta\right)\right\}^{2}} .
$$

Taking $K=\frac{C_{7}}{k_{0} c_{0}}$, we can have $G^{i \bar{j}} \partial_{z_{i}^{\prime} \bar{z}_{j}} h \geq 0$ in $\psi(B(0, \delta) \bigcap \Omega)$. By the maximum principle, we know $h\left(z^{\prime}\right)$ achieves its maximum at $z^{\prime}=0$. Hence $\left.h\left(z^{\prime}\right)\right|_{z^{\prime}=\psi(z)}$ achieves its maximum at $z_{0}=0$. Thus

$$
0 \leq D_{v} h(0) \leq D_{v v} u\left(z_{0}\right)+C_{8}
$$

So we have $D_{v v} u\left(z_{0}\right) \geq-C_{8}$.

The same argument for

$$
h_{1}\left(z^{\prime}\right)=4 \operatorname{Re}\left[\left\langle\partial_{z^{\prime}} u, \partial_{z^{\prime}} r_{0}\right\rangle-\sum_{j=1}^{n-1} A_{j} \partial_{z_{j}^{\prime}} u\right]-\phi\left(z^{\prime}, u\right)-\left.K r(z)\right|_{z=\psi^{-1}\left(z^{\prime}\right)}+K_{1} \operatorname{Re}\left(z_{n}^{\prime}\right)
$$

can give

$$
D_{v v} u\left(z_{0}\right) \leq C_{9} .
$$

This completes the estimates of the double normal derivative on the boundary.

\subsection{Estimate of global second derivatives}

Theorem 4.2. Suppose $\Omega \subset \mathbb{C}^{n}$ is a $C^{4}$ strictly pseudoconvex domain satisfying (1.3) and $\varphi \in$ $C^{3}(\partial \Omega)$. Let $\Theta(z) \in C^{2}(\bar{\Omega})$ with $\frac{(n-2) \pi}{2}<\Theta(z)<\frac{n \pi}{2}$ in $\bar{\Omega}$ and $u \in C^{4}(\Omega) \cap C^{3}(\bar{\Omega})$ be a solution 
of special Lagrangian equation (1.2), then we have

$$
\sup _{\bar{\Omega}}\left|D^{2} u\right| \leq M_{2}
$$

where $M_{2}$ depends on $n, \Omega, 2 \kappa_{\min }+\varepsilon, \max _{\bar{\Omega}} \Theta, \min _{\bar{\Omega}} \Theta,|u|_{C^{1}},|\Theta|_{C^{2}}$ and $|\varphi|_{C^{3}}$.

Proof. From (2.2c), we know

$$
\Delta u=4 \sum_{i=1}^{n} u_{i \bar{i}}=4\left(\lambda_{1}+\cdots+\lambda_{n}\right)>0
$$

By the argument in Li's [19], we know that we only need to prove that

$$
D_{\zeta \zeta} u(z) \leq M_{2}, \quad \forall(z, \zeta) \in \bar{\Omega} \times \mathbb{S}^{2 n-1} .
$$

As the real case in [23], we use the auxiliary function

$$
Q(z, \zeta)=D_{\zeta \zeta} u-v(z, \zeta)+K_{1}|z|^{2}+|D u|^{2},
$$

where $v(z, \zeta)=2\langle\zeta, v\rangle\left\langle\zeta^{\prime}, D \varphi-\varepsilon D u-D_{l} u D v^{l}\right\rangle=a_{l} D_{l} u+b, v=\left(v^{1}, v^{2}, \cdots, v^{2 n}\right) \in \mathbb{S}^{2 n-1}$ is a $C^{3}(\bar{\Omega})$ extension of the outer unit normal vector field on $\partial \Omega$

$$
\zeta^{\prime}=\zeta-\langle\zeta, v\rangle v, \quad a_{l}=-2\langle\zeta, v\rangle\left\langle\zeta^{\prime}, D v^{l}\right\rangle-2 \varepsilon\langle\zeta, v\rangle\left(\zeta^{\prime}\right)^{l}, \quad b=2\langle\zeta, v\rangle\left\langle\zeta^{\prime}, D \varphi\right\rangle,
$$

and $K_{1}>0$ is to be determined later.

For any $z \in \Omega$, we rotate the coordinates such that $\left\{\partial_{i j} u(z)\right\}$ is diagonal with $\lambda_{i}=u_{i \bar{i}}$ and $\lambda_{1} \geq \lambda_{2} \geq \cdots \geq \lambda_{n}$. Then we have

$$
F^{i \bar{j}}=: \frac{\partial \arctan \partial \bar{\partial} u}{\partial u_{i \bar{j}}}= \begin{cases}\frac{1}{1+\lambda_{i}^{2}}, & \text { if } i=j, \\ 0, & \text { if } i \neq j .\end{cases}
$$

Hence we can get from Property 2.1

$$
\begin{aligned}
& F^{1 \overline{1}} \leq F^{2 \overline{2}} \leq \cdots \leq F^{n \bar{n}}, \\
& F^{n \bar{n}}=\frac{1}{1+\lambda_{n}^{2}} \geq c_{0}>0, \\
& F^{i \bar{i}} u_{i \bar{i}}=\frac{\lambda_{i}}{1+\lambda_{i}^{2}} \in\left(-\frac{1}{2}, \frac{1}{2}\right),
\end{aligned}
$$

where $c_{0}=\frac{1}{1+\max \left\{\tan \left(\frac{(n-1) \pi}{2}-\min _{\bar{\Omega}} \Theta\right), \tan \left(\frac{1}{n} \max _{\bar{\Omega}} \Theta\right)\right\}^{2}}$. 
For any fixed $\zeta \in \mathrm{S}^{n-1}$, we have

$$
\begin{aligned}
F^{i \bar{j}} \partial_{i \bar{j}} Q(z, \zeta)= & F^{i \bar{j}} \partial_{i \bar{j}} D_{\zeta \zeta} u-F^{i \bar{i}}\left[\partial_{i \bar{i}} a_{k} D_{k} u+\partial_{\bar{i}} a_{k} \partial_{i} D_{k} u+\partial_{i} a_{k} \partial_{\bar{i}} D_{k} u+a_{k} \partial_{i \bar{i}} D_{k} u+\partial_{i \bar{i}} b\right] \\
& +2 F^{i \bar{i}} \partial_{i} D_{k} u \partial_{\bar{i}} D_{k} u+2 D_{k} \Theta D_{k} u+K_{1} \sum_{i=1}^{n} F^{i \bar{i}} \\
\geq & D_{\zeta \zeta} \Theta-A\left|D_{\zeta} \Theta\right|^{2}-\left[\left|D^{2} a_{k}\right||D u|+\left|D^{2} b\right|\right] \sum_{i=1}^{n} F^{i \bar{i}}-F^{i \bar{i}}\left(\partial_{\bar{i}} a_{k} \partial_{i} D_{k} u+\partial_{i} a_{k} \partial_{\bar{i}} D_{k} u\right) \\
& -\left|a_{k} D_{k} \Theta\right|+2 F^{i \bar{i}} \partial_{i} D_{k} u \partial_{\bar{i}} D_{k} u-2\left|D_{k} \Theta D_{k} u\right|+K_{1} \sum_{i=1}^{n} F^{i \bar{i}} .
\end{aligned}
$$

It is easy to know

$$
\begin{aligned}
& \sum_{i=1}^{n} F^{i \bar{i}}>F^{n \bar{n}} \geq c_{0}, \\
& 2 F^{i \bar{i}} \partial_{i} D_{k} u \partial_{\bar{i}} D_{k} u-F^{i \bar{i}}\left(\partial_{\bar{i}} a_{k} \partial_{i} D_{k} u+\partial_{i} a_{k} \partial_{\bar{i}} D_{k} u\right) \geq-F^{i \bar{i}} \partial_{i} a_{k} \partial_{\bar{i}} a_{k} .
\end{aligned}
$$

Choose

$$
K_{1}=2\left[\left|D^{2} a_{k}\right||D u|+\left|D^{2} b\right|\right]+\frac{2\left[\left|D^{2} \Theta\right|+A|D \Theta|^{2}+\left|a_{k}\right||D \Theta|+2|D \Theta||D u|\right]}{c_{0}},
$$

then we can get

$$
F^{i \bar{j}} \partial_{i j} Q(z, \zeta) \geq 0
$$

So $\max _{\bar{\Omega}} Q(z, \zeta)$ attains its maximum on $\partial \Omega$. Hence $\max _{\bar{\Omega} \times \mathrm{S}^{2 n-1}} Q(z, \zeta)$ attains its maximum at some point $z_{0} \in \partial \Omega$ and some direction $\zeta_{0} \in \mathbb{S}^{2 n-1}$.

Then we continue our proof in the following two cases following the idea of [19].

(a) If $\zeta_{0}$ is non-tangential at $z_{0} \in \partial \Omega$, then we can write $\zeta_{0}=\alpha \tau+\beta \nu$, where $\tau \in \mathbb{S}^{2 n-1}$ is tangential at $z_{0}$, that is $\langle\tau, v\rangle=0, \alpha=\left\langle\zeta_{0}, \tau\right\rangle, \beta=\left\langle\zeta_{0}, v\right\rangle \neq 0$, and $\alpha^{2}+\beta^{2}=1$. Then we have

$$
\begin{aligned}
& D_{\zeta_{0} \zeta_{0}} u\left(z_{0}\right) \\
= & \alpha^{2} D_{\tau \tau} u\left(z_{0}\right)+\beta^{2} D_{v v} u\left(z_{0}\right)+2 \alpha \beta D_{\tau v} u\left(z_{0}\right) \\
= & \alpha^{2} D_{\tau \tau} u\left(z_{0}\right)+\beta^{2} D_{v v} u\left(z_{0}\right)+2\left(\xi_{0} \cdot v\right)\left[\xi_{0}-\left(\xi_{0} \cdot v\right) v\right] \cdot\left[D \varphi-\varepsilon D u-D_{l} u D v^{l}\right],
\end{aligned}
$$

hence

$$
Q\left(z_{0}, \zeta_{0}\right)=\alpha^{2} Q\left(z_{0}, \tau\right)+\beta^{2} Q\left(z_{0}, v\right) .
$$

From the definition of $Q\left(z_{0}, \zeta_{0}\right)$, we know

$$
Q\left(z_{0}, \zeta_{0}\right) \leq Q\left(z_{0}, v\right) \leq C_{10}
$$


and we can prove (4.14).

(b) If $\xi_{0}$ is tangential at $z_{0} \in \partial \Omega$, then we have

$$
\begin{aligned}
0 & \leq D_{v} Q\left(z_{0}, \zeta_{0}\right)=D_{v} D_{\zeta_{0} \zeta_{0}} u-D_{v} a_{l} D_{l} u-a_{l} D_{v} D_{l} u-D_{v} b+2 D_{k} u D_{v} D_{k} u+K_{1} D_{v}|z|^{2} \\
& \leq D_{v} D_{\zeta_{0} \zeta_{0}} u+\left[2 D_{k} u-a_{k}\right] D_{v} D_{k} u+C_{11} .
\end{aligned}
$$

By the boundary condition, we know

$$
\begin{aligned}
D_{v} D_{\zeta_{0} \zeta_{0}} u & =D_{\zeta_{0} \zeta_{0}} D_{v} u-\left(D_{\zeta_{0} \zeta_{0}} v^{k}\right) D_{k} u-2\left(D_{\zeta_{0}} v^{k}\right) D_{\zeta_{0}} D_{k} u \\
& =D_{\zeta_{0} \zeta_{0}}(-\varepsilon u+\varphi)-\left(D_{\zeta_{0} \zeta_{0}} v^{k}\right) D_{k} u-2\left(D_{\zeta_{0}} v^{k}\right) D_{\zeta_{0}} D_{k} u \\
& \leq-\varepsilon Q\left(z_{0}, \zeta_{0}\right)+C_{12}-2\left(D_{\zeta_{0}} v^{k}\right) D_{\zeta_{0}} D_{k} u
\end{aligned}
$$

Following the argument of [19], we can get

$$
\begin{aligned}
& \left|D_{v} D_{k} u\right| \leq C_{13}, \\
& -2\left(D_{\zeta_{0}} v^{k}\right) D_{\zeta_{0}} D_{k} u \leq-2 \kappa_{\min } Q\left(z_{0}, \zeta_{0}\right)+C_{14} .
\end{aligned}
$$

From (1.3), it holds $2 \kappa_{\min }+\varepsilon>0$. So

$$
Q\left(z_{0}, \zeta_{0}\right) \leq \frac{C_{11}+C_{12}+\left(2|D u|+\left|a_{k}\right|\right) C_{13}+C_{14}}{2 \kappa_{\min }+\varepsilon} .
$$

Then we can easily get (4.14).

Remark 4.1. As the discussions in Remark 3.1, to prove Theorem 1.2, we need to consider the complex special Lagrangian equation (1.2) with $\varepsilon \rightarrow 0$. Under the assumptions of Theorem 1.2, and for any sufficiently small $\varepsilon$, we can obtain the following proof of Theorem 4.1 and Theorem 4.2,

$$
\sup _{\bar{\Omega}}\left|D^{2} u^{\varepsilon}\right|=\sup _{\bar{\Omega}}\left|D^{2}\left(u^{\varepsilon}-\frac{1}{|\Omega|} \int_{\Omega} u^{\varepsilon}\right)\right| \leq \widetilde{M}_{2}
$$

where $\widetilde{M}_{2}$ depends on $n, \Omega, \max _{\bar{\Omega}} \Theta, \min _{\bar{\Omega}} \Theta,|\Theta|_{C^{2}}$ and $|\varphi|_{C^{3}}$, and is independent of $\varepsilon$.

\section{Existence of the boundary problems}

In this section we complete the proofs of the Theorem 1.1 and Theorem 1.2.

\subsection{Proof of Theorem 1.1}

For the Neumann problem of complex special Lagrangian equation (1.2), we have established the $C^{0}, C^{1}$ and $C^{2}$ estimates in Section 2, Section 3, Section 4 , respectively. By the global $C^{2}$ priori estimate, the special Lagrangian equation (1.2) is uniformly elliptic in $\bar{\Omega}$. 
From the concavity Lemma (Lemma 2.2 in [8]), we know $-e^{-A \arctan \partial \bar{\partial} u}$ is concave with respect to $\partial \bar{\partial} u$, where $A$ is defined in Property 2.2. Following the discussions in [22], we can get the global Hölder estimates of second derivative,

$$
|u|_{C^{2, \alpha}(\bar{\Omega})} \leq C,
$$

where $C$ and $\alpha$ depend on $n, \Omega, \max _{\bar{\Omega}} \Theta, \min _{\bar{\Omega}} \Theta,|\Theta|_{C^{2}}$ and $|\varphi|_{C^{3}}$. From (5.1), one also obtains $C^{3, \alpha}(\bar{\Omega})$ estimates by differentiating Eq. (1.2) and applies the Schauder theory for linear uniformly elliptic equations.

Applying the method of continuity (see [21]), the existence of the classical solution holds. By the standard regularity theory of uniformly elliptic partial differential equations, we can obtain the higher regularity.

\subsection{Proof of Theorem 1.2}

By a similar proof of Theorem 1.1, we know there exists a unique solution $u^{\varepsilon} \in C^{3, \alpha}(\bar{\Omega})$ to (1.2) for any small $\varepsilon>0$. Let $v^{\varepsilon}=u^{\varepsilon}-\frac{1}{|\Omega|} \int_{\Omega} u^{\varepsilon}$, and it is easy to know that $v^{\varepsilon}$ satisfies

$$
\begin{cases}\arctan \partial \bar{\partial} v^{\varepsilon}=\Theta(z) & \text { in } \Omega \\ D_{v}\left(v^{\varepsilon}\right)=-\varepsilon v^{\varepsilon}-\frac{1}{|\Omega|} \int_{\Omega} \varepsilon u^{\varepsilon}+\varphi(z) & \text { on } \partial \Omega\end{cases}
$$

By the global gradient estimate (3.12), it is easy to know $\varepsilon \sup \left|D u^{\varepsilon}\right| \rightarrow 0$. Hence there is a constant $\beta$ and a function $v \in C^{2}(\bar{\Omega})$, such that

$$
-\varepsilon \mathcal{U}^{\varepsilon} \rightarrow \beta, \quad-\varepsilon v^{\varepsilon} \rightarrow 0, \quad-\frac{1}{|\Omega|} \int_{\Omega} \varepsilon u^{\varepsilon} \rightarrow \beta, \quad v^{\varepsilon} \rightarrow v,
$$

uniformly in $C^{2}(\bar{\Omega})$ as $\varepsilon \rightarrow 0$. It is easy to verify that $v$ is a solution of

$$
\begin{cases}\arctan \partial \bar{\partial} v=\Theta(z) & \text { in } \Omega \\ D_{v} v=\beta+\varphi(z) & \text { on } \partial \Omega\end{cases}
$$

If there is another function $v_{1} \in C^{2}(\bar{\Omega})$ and another constant $\beta_{1}$ such that

$$
\begin{cases}\arctan \partial \bar{\partial} v_{1}=\Theta(z) & \text { in } \Omega, \\ D_{v}\left(v_{1}\right)=\beta_{1}+\varphi(z) & \text { on } \partial \Omega\end{cases}
$$

Applying the maximum principle and Hopf Lemma, we can know $\beta=\beta_{1}$ and $v-v_{1}$ is a constant. By the standard regularity theory of uniformly elliptic partial differential equations, we can obtain the higher regularity. 


\section{Acknowledgements}

Research of the first author was supported by ZJNSF No. LY17A010022 and NSFC No. 11771396. Research of the second author and third author was supported by NSFC No. 11471188 and Wu Wen-Tsun Key Laboratory of Mathematics in USTC. Research of the third author was supported by China Scholarship Council.

\section{References}

[1] E. Bedford and J. E. Fornaess, Counterexamples to regularity for the complex MongeAmpère equation, Invent. Math., 50 (1979), 129-134.

[2] L. Caffarelli, L. Nirenberg and J. Spruck, The Dirichlet problem for nonlinear second order elliptic equations I, Monge-Ampère equations, Commun. Pure Appl. Math., 37 (1984), 369402.

[3] L. A. Caffarelli, J. J. Kohn, L. Nirenberg and J. Spruck, The Dirichlet problem for nonlinear second-order elliptic equations, II. Complex Monge-Ampère and uniformly elliptic equations, Commun. Pure Appl. Math., 38 (1985), 209-252.

[4] L. Caffarelli, L. Nirenberg and J. Spruck. The Dirichlet problem for nonlinear second order elliptic equations III, Functions of the eigenvalues of the Hessian, Acta Math., 155 (1985), 261-301.

[5] C. Q. Chen, X. N. Ma and W. Wei, The Neumann problem of special Lagrangian equations with supercritical phase, Preprint, 2017.

[6] C. Q. Chen and D. K. Zhang, The Neumann problem for Hessian quotient equations, Preprint, 2016.

[7] Y. Z. Chen and L. C. Wu, Second Order Elliptic Equations and Elliptic Systems, Am. Math. Soc., Providence, RI, 1998.

[8] T. C. Collins, S. Picard and X. Wu, Concavity of the Lagrangian phase operator and applications, Calc. Var., 56 (2017), 89.

[9] S. Dinew and S. Kolodziej, Liouville and Calabi-Yau type theorems for complex Hessian equations, Am. J. Math., 139(2) (2017), 403-415.

[10] J. X. Fu and S. T. Yau, The theory of superstring with flux on non-Kähler manifolds and the complex Monge-Ampère equation, J. Diff. Geom., 78(3) (2008), 369-428.

[11] D. Gilbarg and N. Trudinger, Elliptic Partial Differential Equations of Second Order, Grundlehren der Mathematischen Wissenschaften, Vol. 224. Springer-Verlag, Berlin-New York, 1977. $x+401$ pp. ISBN: 3-540-08007-4.

[12] B. Guan, The Dirichlet problem for complex Monge-Ampère equations and regularity of the pluri-complex Green function, Comm. Anal. Geom., 6 (1998), 687-703; a correction, 8 (2000), 213-218.

[13] R. Harvey and B. Lawson, Calibrated geometries, Acta Math., 148(1) (1982), 47-157.

[14] Z. L. Hou, X. N. Ma and D. M. Wu, A second order estimate for complex Hessian equations on a compact Kähler manifold, Math. Res. Lett., 17 (2010), 547-561.

[15] N. Ivochkina, Solutions of the Dirichlet problem for certain equations of Monge-Ampère type (in Russian), Mat. Sb., 128 (1985), 403-415: English translation in Math. USSR Sb., 56 (1987).

[16] F. Jiang and N. S. Trudinger, Oblique boundary value problems for augmented Hessian equations I, Bull. Math. Sci., 8 (2018), 353-411. 
[17] F. Jiang and N. S. Trudinger, Oblique boundary value problems for augmented Hessian equations II, Nonlinear Anal. Theory Methods Appl., 154 (2017), 148-173.

[18] S. Kolodziej and N.-C. Nguyen, Weak solutions of complex Hessian equations on compact Hermitian manifolds, Compos. Math., 152 (2016), 2221-2248.

[19] S. Y. Li, On the Neumann problems for Complex Monge-Ampère equations, Indiana Univ. Math. J., 43 (1994), 1099-1122.

[20] S. Y. Li, On the Dirichlet problems for symmetric function equations of the eigenvalues of the complex Hessian, Asian J. Math., 8 (2004), 087-106.

[21] G. Lieberman, Oblique Boundary Value Problems for Elliptic Equations, World Scientific Publishing, (2013).

[22] G. Lieberman and N. Trudinger, Nonlinear oblique boundary value problems for nonlinear elliptic equations, Trans. Amer. Math. Soc., 295 (1986), 509-546.

[23] P. L. Lions, N. Trudinger and J. Urbas, The Neumann problem for equations of MongeAmpère type, Commun. Pure Appl. Math., 39 (1986), 539-563.

[24] X. N. Ma and G. H. Qiu, The Neumann Problem for Hessian Equations, Commun. Math. Phys., 366 (2019), 1-28.

[25] D. H. Phong, S. Picard and X. W. Zhang, The Fu-Yau equation with negative slope parameter, Invent. Math., 209 (2017), 541-576.

[26] A. Stromginer, S.-T. Yau and E. Zaslow, Mirror symmetry is T-duality, Nuclear Phys. B, 479 (1996), 243-259.

[27] N. S. Trudinger, On the Dirichlet problem for Hessian equations, Acta Math., 175 (1995), 151-164.

[28] D. K. Wang and Y. Yuan, Hessian estimates for special Lagrangian equations with critical and supercritical phases in general dimensions, Amer. J. Math., 136 (2014), 481-499.

[29] M. Warren and Y. Yuan, Hessian and gradient estimates for three dimensional special Lagrangian equations with large phase, Amer. J. Math., 132 (2010), 751-770. 